\title{
On the Multiplexing of Data and Metadata for Ultra-Reliable Low-Latency Communications in 5G
}

\author{
Ali Karimi, Member, IEEE, Klaus I. Pedersen, Senior member, IEEE, Nurul Huda Mahmood, Member, IEEE, \\ Gilberto Berardinelli, Member, IEEE, and Preben Mogensen, Member, IEEE
}

\begin{abstract}
This paper addresses the problem of downlink radio resource management for ultra-reliable low-latency communications (URLLC) in fifth generation (5G) systems. To support low-latency communications, we study performance of two multiplexing schemes namely in-resource control signalling and joint encoding of data and metadata. In the former, the metadata and data are separately encoded and the metadata is sent at the beginning of transmission time prior to the data. Thus, it benefits from a low-complexity receiver structure to decode the data block. The latter takes advantages of transmitting a larger blocklength to enhance the reliability and improve spectrum efficiency by jointly encoding data and metadata as a single codeword. Dealing with small URLLC payloads, the overhead and error of sending metadata are not negligible and have a significant impact on the system performance in terms of resource usage the reliability of transmission. For each scheme, we derive expressions for the outage probability and resource usage by taking into account impacts of the finite blocklength payloads, overhead and error of sending metadata, and probability of error in uplink feedback channel. We propose a novel framework for joint data and metadata link adaptation to minimize the average number of allocated resources, while ensuring the stringent URLLC quality of service requirements. An optimization problem is formulated for each scheme that is mixed-integer nonconvex problem, difficult to solve in polynomial time. Solutions based on successive convex optimization are proposed. Numerical evaluations show that the proposed algorithms perform close to the optimal solution and demonstrate remarkable gains of up to $27 \%$ improvement in resource usage. Finally, we present sensitivity analysis of the results for various network parameters.
\end{abstract}

Index Terms-URLLC, 5G, Radio resource management, Control channel information, Link adaptation.

\section{INTRODUCTION}

Recently, third generation partnership project (3GPP) has introduced the first release of fifth generation New Radio (5G NR) [1]. Unlike Long-Term Evolution (LTE) networks, which are primarily serving mobile broadband (MBB) and machine type communications (MTC) services, 5G NR is designed to

Copyright (c) 2015 IEEE. Personal use of this material is permitted. However, permission to use this material for any other purposes must be obtained from the IEEE by sending a request to pubs-permissions@ieee.org.

A. Karimi is with Nokia Bell Labs, Munich 81541, Germany (e-mail: ali.karimidehkordi@nokia.com).

G. Berardinelli is with the Wireless Communication Networks (WCN) Section, Department of Electronic Systems, Aalborg University, Aalborg 9220, Denmark (e-mail: gb@es.aau.dk).

N. H. Mahmood is with the Centre for Wireless Communications, University of Oulu, Oulu 90014, Finland (e-mail: NurulHuda.Mahmood@oulu.fi).

K. I. Pedersen and P. Mogensen are with Nokia Bell labs, Aalborg 9220, Denmark, and also with the WCN Section, Aalborg University, Aalborg 9220, Denmark (e-mails: klaus.pedersen@nokia-bell-labs.com; pm@es.aau.dk). additionally support ultra-reliable low-latency communications (URLLC) [2], [3]. As the name suggests, URLLC target applications requiring high reliability with low-latency for emerging use cases like industrial automation, intelligent transport systems, and haptic communications [4]-[6]. A typical URLLC target is one-way reliability of $99.999 \%$ for a data unit of 32 bytes to be delivered within a tight time budget of one millisecond (msec) [2], [7].

\section{A. Brief Overview of the State of the Art on URLLC}

In recent years, extensive research efforts have been made to enable URLLC in 5G NR. As highlighted in [8], current LTE networks have not been designed to support the stringent URLLC requirements. As one of the main building blocks to reduce the latency, flexible frame structure and user scheduling over short transmission time intervals (TTIs) are discussed in [9]. Performance analysis of URLLC through advanced system-level simulations are investigated for the downlink (DL) and uplink (UL) transmission directions in [10] and [11] respectively. Multi-user multiplexing solutions for the coexistence of URLLC and enhanced MBB (eMBB) traffic are presented [12]-[16]. The works in [17] and [18] study centralized radio access network (C-RAN) architecture and dynamic point selection to reduce the obstructive queuing delay of URLLC payloads. Reliability enhancement by means of data packet duplication is presented in [19], [20]. To overcome the timely handshaking procedures of grant-based scheduling in UL, the studies in [21] and [22] investigate grant free and semi-grant free access protocols, respectively. UL multi-cell reception design has been extensively addressed in [23] by comparing the achievable capacity of various receivercombining techniques. Several studies explore URLLC for vehicular communications [24]-[29].

To enhance spectral efficiency and enable massive connectivity, network design through non-orthogonal multiple access (NOMA) for URLLC is investigated in [30]-[32]. Performance comparison of orthogonal multiple access (OMA) and NOMA is provided in [33]. The study in [34] presents a hybrid channel access solution based on machine learning techniques. The potential of New Radio Unlicensed spectrum (NR-U) for URLLC is discussed in [35]. The authors in [36] focus on mobile edge computing and user/server association to ensure low-latency communication. Finally, different cooperative protocols and resource allocation schemes for URLLC have been extensively considered in [37]-[41].

As URLLC mainly entails transmission of small payloads, applying the well-known Shannon's Capacity under asymmet- 
ric assumptions (which is valid when the codeword size tends to infinity) is not an appropriate approach for radio resource allocation and performance evaluation [42], [43]. Informationtheoretic principles of finite blocklength (FBL) communications are studied in [44], [45]. It is shown that applying the law of large numbers for averaging channel distortions and noise is not applicable for FBL packet transmissions. The achievable rate is subject to a rate penalty from the Shannon's Capacity which is proportional to the square root of encoded blocklength [45], [46].

Taking into account the results from FBL communication theory, many researches have studied several radio resource management techniques and investigated various URLLC enablers to further boost the 5G performance. Particularly, optimal power allocation and subcarrier assignment policies for DL multi-user scenarios are proposed in [47] and [48], respectively. The authors in [49] adopt multi-class queuing theory to design and analyse the network performance of URLLC. The studies in [50] and [51] investigate effective capacity for FBL regime and propose a bandwidth assignment policy for joint UL and DL transmission to guarantee end-toend (E2E) latency.

As a well-known technique to enhance the reliability and spectral efficiency, throughput analysis of FBL hybrid automatic repeat request (HARQ) is investigated in [52]. Triggered by [52], several works have looked at various HARQ retransmission protocols for FBL communications (see e.g. [53]-[57]). Interesting power allocation ideas are proposed to minimize the energy consumption of Chase-Combining HARQ (CC-HARQ) for Rayleigh and Nakagami-m blockfading channels in [53] and [54], respectively. On the other hand, resource allocation schemes for incremental redundancy HARQ (IR-HARQ) are discussed in [55], [56]. However, due to the tight latency targets of URLLC applications and the time requirement of HARQ procedures, HARQ performance is limited to usually one retransmission for URLLC [58], [59].

\section{B. Motivation and Main Contributions}

Successful data communication in DL is conditioned on the reliable transmission of metadata (also referred to as control channel information or scheduling grant). One common assumption of many studies is that the metadata has negligible impact on the system performance. This perspective is generally true for analysing eMBB where large data blocks are usually scheduled with aggressive modulation and coding schemes (MCSs) to maximize the throughput [60].

However, the situation is different for URLLC cases as the data payloads are small, with size comparable to the metadata, and transmissions are subject to tight reliability and latency constraints [59], [61]. While most of the existing contributions have mainly focused on various aspects of data scheduling (see e.g. [47]-[53]), only a few studies have addressed technical issues that arise from metadata transmission. In one example, it is indicated in [62], [63] that for URLLC a considerable proportion of the network resources are used for metadata allocation. Recent studies in [59], [64] take error probability of decoding metadata into account and derive bounds of reliability interdependences between data and metadata. In general, low-error transmission of metadata is essential to support URLLC. At the same time, improving the reliability by encoding with more conservative MCSs leads to additional resource usage and increases the probability of queuing [50]. Due to these tradeoffs, a new link adaptation design is required to efficiently manage joint resource allocation for both data and metadata while ensuring fulfilments of URLLC requirements.

Besides the challenges that arise from joint data and metadata allocation, the probability of error in the UL feedback channel is another important issue that has significant impact on the URLLC performance [65], [66]. On one hand, erroneous decoding/detection of negative-acknowledgement (NACK) or discontinuous transmission (DTX) as acknowledgement $(\mathrm{ACK})$ results in outage latency for URLLC services. On the other hand, conservative decoding of feedback signals (e.g. asymmetric decoding of ACK as NACK or DTX) causes redundant retransmissions and contributes to additional resource usage.

Motivated by the above discussions, in this paper, we propose novel solutions for the problem of URLLC DL radio resource allocation by taking into account non-ideal metadata and feedback transmissions. We start by exploring fundamentals of data and metadata multiplexing in wireless channels. Two multiplexing schemes, namely in-resource control signalling [9] and joint encoding of data and metadata [61], are studied. The main idea of the former is to separately allocate the metadata at the beginning of the DL sub-frame. Thereby, it benefits from low-computational complexity for decoding data as well as reduced processing time and may enable opportunity for fast HARQ feedback [67], [68]. The latter achieves the enhanced reliability and spectral efficiency gains of transmitting larger blocklength by jointly encoding data and metadata in a single codeword.

For each scheme, we adopt the FBL communication theory and derive expressions for the outage probability and resource usage. The impacts of overhead/error of sending metadata and non-ideal feedback signals are explicitly considered. A resource allocation problem is formulated for each scheme. Specially, we aim for jointly optimizing link adaptation for metadata and data to minimize total resource usage subject to URLLC constraints. To the best of our knowledge, these problems have not been investigated in the existing literature. The problems are mixed-integer non-linear optimizations that are difficult to solve in polynomial time. To overcome the non-convexity of problems, we propose solutions based on successive convex optimization. Numerical results indicate that the proposed algorithms significantly improve resource efficiency and achieve near-optimal solutions. Finally, we provide extensive numerical results and complementary discussions to investigate impacts of different network parameters on the solution's performance.

In summary, key contributions of this paper are the followings:

- We introduce a new framework for the analysis of URLLC performance which includes different multiplexing schemes of data and metadata. 
TABLE I: List of Symbols

\begin{tabular}{ll}
\hline Symbol & Definition \\
\hline General & symbols \\
$M$ & Metadata size [byte] \\
$D$ & Data size [byte] \\
$\gamma$ & Signal to noise ratio (SNR) \\
$P_{\text {out }}^{t a r}$ & Outage probability target \\
$P_{e}^{a m}$ & Probability of decoding ACK as DTX \\
$P_{e}^{m a}$ & Probability of decoding DTX as ACK \\
$P_{e}^{n a}$ & Probability of decoding NACK as ACK \\
$P_{e}^{a n}$ & Probability of decoding ACK as NACK \\
$\mathbb{R}^{a n}$ & Real numbers \\
$\mathbb{R}^{+}$ & Positive real numbers \\
$\mathbb{N}^{+}$ & Positive integer numbers \\
In-resource control signalling \\
$d_{i}$ & Data blocklength in $i$-th transmission \\
$P_{e}^{d_{i}}$ & Data block error rate probability (BLEP) in $i$-th transmission \\
$P_{e}^{d_{12}}$ & Data BLEP upon HARQ retransmission \\
$m_{i}$ & Metadata blocklength in $i$-th transmission \\
$P_{e}^{m_{i}}$ & Metadata BLEP in $i$-th transmission \\
$N^{I t}$ & Average number of resource usage \\
$P_{\text {out }}^{I t}$ & Overall outage probability \\
Joint encoding of data and metadata \\
$n_{i}$ & Codeword length in $i$-th transmission \\
$P_{e}^{J_{i}}$ & BLEP in $i$-th transmission \\
$N^{J t}$ & Average number of resource usage \\
$P_{\text {out }}^{J t}$ & Overall outage probability \\
\hline
\end{tabular}

- We present a novel approach for optimizing joint link adaptation for data and metadata. To address this, we propose solutions based on successive convex optimization.

- Finally, we provide an extensive set of simulation results to evaluate the performance of the proposed solutions under different network settings and scheduling scenarios. It is shown that the proposed dynamic link adaptation algorithms offer remarkable gains and reduce resource consumption.

The rest of this paper is organized as follows. The system model and basic assumptions are described in Section II. In Section III and IV, we discuss the problem formulation and present the solution, respectively. Numerical results are provided in Section V. Finally, Section VI concludes the paper. Table I includes a list of the main symbols used in this paper.

\section{System Model And Basic TRANSmission ASSUMPTIONS}

We focus on DL performance assuming orthogonal frequency division multiple access (OFDMA) transmission in which a base station (BS) serves user-equipments (UEs) with packets of $D$ bytes. URLLC requires a very low outage probability target of $P_{\text {out }}^{t a r}$ within one msec latency. To reduce the transmission time and achieve such tight latency requirement, we adopt 5G NR flexible numerology with minislot scheduling. Assuming a mini-slot length of two to four OFDM symbols with $15-30 \mathrm{kHz}$ sub-carrier spacing and by taking into account the packet transmission/processing times, this leaves enough time budget for a single retransmission (if the initial transmission fails) [58], [59], [69].

Transmitting $D$ bytes of data requires preceding transmission of $M$ bytes of metadata carrying transceiver/transmission specific information such as device-ID, adopted MCS, applied precoding matrix, allocated physical resource blocks, etc. Two proposals are investigated for multiplexing of data and metadata. In-resource control signalling along with front-loading of demodulation reference signals is proposed in [8], [9]. Following 5G NR user-centric design for dynamic scheduling of URLLC payloads, whenever the network schedules a UE, the corresponding control information is separately encoded and sent at the beginning of the transmitted packet.

The second approach, joint encoding/decoding of data and metadata is proposed in [61], [70] aiming to reduce error probability and spectrum inefficiency initiated by FBL codewords. The main idea is to combine metadata and data in a single packet of size $M+D$ bytes. It is shown that transmission with relatively larger blocklength is more reliable and spectrally efficient [45]. However, this concept suffers from high computational-complexity as the UE is required to decode all the messages, despite if it was not the intended destination. Thus, it is a trade-off between spectral efficiency and complexity (additional UE processing time and energy).

In line with [6], [51], [71]-[74], we analyse URLLC performance using the FBL theory in quasi-static flat fading channels [45]. That is, for a payload of $b$ bits information mapped to a codeword of length $n$ channel uses, decoding error probability $\epsilon$ is approximated as

$$
\epsilon \approx E(n, \gamma, b) \triangleq Q\left(\frac{n C(\gamma)-b}{\sqrt{n V(\gamma)}}\right),
$$

where $C(\gamma)=\log _{2}(1+\gamma)$ is the Shannon capacity of complex channels for a given SNR $\gamma . Q(\cdot)$ is the Gaussian Q-function $\left(Q(x)=\frac{1}{\sqrt{2 \pi}} \int_{x}^{\infty} \exp \left(-\frac{u^{2}}{2}\right) \mathrm{d} u\right)$ and $V(\gamma)=$ $\frac{1}{\ln ^{2} 2}\left(1-\frac{1}{(1+\gamma)^{2}}\right)$ is channel dispersion factor [45]. One can apply (1) with different channel dispersion factor for performance analysis of non-Gaussian interference channels [75], [76]. However, applying this does not change the problem formulation and the provided solution of this paper. Performing periodic channel state information (CSI) measurements, we assume that the CSI knowledge is available at both transceivers [33], [47], [48]. CSI acquisition procedure is run in parallel with packet scheduling and tight resolution periodicity (e.g. every two msec) to increase the measurement accuracy. Thus, whenever a packet arrives at the network, required CSI is already available and this would not impose additional latency for URLLC services [10], [13], [62]. As the URLLC latency target is smaller than the channel coherence time in most of the applications, it is assumed that the channel remains constant during the initial transmission and the potential retransmission [52], [55], [68].

Using (1), the minimum blocklength $n^{\min }$ satisfying the outage probability $\epsilon_{0}$ is related to the SNR and the payload size, which can be expressed as

$$
f\left(n^{\min }, \gamma, b, \epsilon_{0}\right) \triangleq n^{\min } C(\gamma)-b-\sqrt{n^{\min } V(\gamma)} Q^{-1}\left(\epsilon_{0}\right)=0,
$$

where $Q^{-1}(\cdot)$ is inverse of the Gaussian Q-function. The function $f(\cdot)$ is convex and has a quadratic form with respect 
to $\sqrt{n^{\text {min }}}$. Solving (2) and after some algebraic manipulations we have

$$
\begin{aligned}
n^{\min }=\frac{b}{C(\gamma)}+ & \frac{\left(Q^{-1}\left(\epsilon_{0}\right)\right)^{2} V(\gamma)}{C^{2}(\gamma)} \times \\
& {\left[1+\left(1+\frac{4 C(\gamma) b}{\left(Q^{-1}\left(\epsilon_{0}\right)\right)^{2} V(\gamma)}\right)^{1 / 2}\right] }
\end{aligned}
$$

\section{PROBlem Formulation}

\section{A. In-Resource Control Signalling}

Assuming separate encoding of metadata and data, the probability of success in the initial transmission is conditioned upon correct decoding of both the metadata and data. Let $m_{i}$ and $d_{i}$ respectively denote the number of allocated resources (i.e., subcarrier symbols) to metadata and data in the $i$-th transmission round $(i \in\{1,2\})$. The number of resources in the initial transmission equals

$$
N^{I_{1}}=m_{1}+d_{1} .
$$

The probability of success $P_{s u c c}^{I_{1}}$ and the corresponding outage probability $P_{\text {out }}^{I_{1}}$ of the first transmission are given by

$$
\begin{aligned}
& P_{\text {succ }}^{I_{1}}=\left(1-P_{e}^{m_{1}}\right)\left(1-P_{e}^{d_{1}}\right), \\
& P_{\text {out }}^{I_{1}}=1-P_{\text {succ }}^{I_{1}}=P_{e}^{d_{1}}+P_{e}^{m_{1}}-P_{e}^{d_{1}} P_{e}^{m_{1}},
\end{aligned}
$$

where $P_{e}^{d_{1}}$ and $P_{e}^{m_{1}}$ denote the BLEP of decoding data and metadata that are scheduled over codeword sizes of $d_{1}$ and $m_{1}$, respectively. After each successful transmission, the UE feeds back an ACK signal to the network. Three possible outcomes of the initial transmission may trigger a retransmission:

1) Decoding $A C K$ as $N A C K$ : It happens when the UE successfully decodes both the metadata and data and sends ACK. But, the ACK message is decoded as NACK at the network. Thereby, an unnecessary HARQ retransmission is scheduled with the same data blocklength $d_{1}$. The received copy is discarded after being decoded by the UE and has no impact on the outage probability. But, it increases the resource utilization by

$$
N_{a n}^{I_{1}}=P_{\text {succ }}^{I_{1}} P_{e}^{a n}\left(m_{2}+d_{1}\right),
$$

where $P_{e}^{a n}$ is the error probability of decoding ACK as NACK.

2) Failure to decode the data: The second case occurs when the UE receives the metadata but fails to decode the data. It then feeds back a NACK. Correct decoding of the NACK by the BS triggers scheduling of the corresponding HARQ retransmission. Otherwise, if the BS decodes the NACK as $\mathrm{ACK}$, it assumes successful transmission and terminates the procedure. This results in latency outage for URLLC applications.

Two retransmission protocols of IR-HARQ and CC-HARQ are considered. Using CC-HARQ, the same codeword as the initial transmission is sent over the retransmission round [54]. The UE combines multiples of received data packets using maximum ratio combining (MRC) to enhance the desired signal power and increase successful decoding probability. With IR-HARQ, data bits are encoded to a parent codeword of length $d L$ channel uses, where $L$ is the maximum number of transmissions [52]. The parent codeword is split into subcodewords of $d$ symbols. A new sub-codeword is consecutively transmitted if the UE fails to correctly decode previous received concatenated samples. The success probability following HARQ retransmission is obtained as [77]

$$
P_{\text {succ }}^{I_{2 a}}=\left(1-P_{e}^{m_{1}}\right)\left(1-P_{e}^{n a}\right)\left(1-P_{e}^{m_{2}}\right)\left(P_{e}^{d_{1}}-P_{e}^{d_{12}}\right),
$$

where $P_{e}^{n a}$ is the error probability of decoding of NACK as ACK. Variable $P_{e}^{d_{12}}$ is the data BLEP after HARQ retransmission combining equals $E\left(d_{1}, 2 \gamma, D\right)$ and $E\left(2 d_{1}, \gamma, D\right)$ for CCHARQ and IR-HARQ, respectively [55], [77]. The additional resource utilization of this case is obtained as

$$
N^{I_{2 a}}=\left(1-P_{e}^{m_{1}}\right) P_{e}^{d_{1}}\left(1-P_{e}^{n a}\right)\left(m_{2}+d_{1}\right) .
$$

Note that $P_{e}^{n a}$ is critical for URLLC services as high values of $P_{e}^{n a}$ prohibit achieving the desired reliability target within the limited time budget. Asymmetric detection to protect NACK signals from being decoded as ACK, NACK repetition, and allocating more resources for feedback signals are among the proposed approaches to enhance the reliability of feedback signals [59], [78], [79].

3) Failure to decode the metadata: In this case, the UE does not know if it is intended to a transmission. Thus, it does not forward any feedback. This is known as discontinuous transmission. When the BS does not receive an ACK/NACK signal by listening to the UL feedback channel, it schedules a new retransmission. Since the control information required to identify the data block in the initial transmission was not correctly decoded, unlike the previous case, there is no possibility of HARQ combining. Accordingly, the probability of success $P_{s u c c}^{I_{2 b}}$ and resource usage $N^{I_{2 b}}$ are driven as

$$
\begin{aligned}
P_{s u c c}^{I_{2 b}} & =P_{e}^{m_{1}}\left(1-P_{e}^{m a}\right)\left(1-P_{e}^{m_{2}}\right)\left(1-P_{e}^{d_{2}}\right), \\
N^{I_{2 b}} & =P_{e}^{m_{1}}\left(1-P_{e}^{m a}\right)\left(m_{2}+d_{2}\right)
\end{aligned}
$$

where $P_{e}^{m a}$ is the probability that the BS erroneously assumes receiving $\mathrm{ACK}$ that leads to an outage.

Following those three error cases for in-resource control signalling, we derive the overall outage probability $P_{\text {out }}^{I t}$ assuming initial transmission plus one retransmission (if needed) as

$$
\begin{aligned}
P_{\text {out }}^{I t}= & 1-P_{\text {succ }}^{I_{1}}-P_{\text {succ }}^{I_{2 a}}-P_{\text {succ }}^{I_{2 b}} \\
= & P_{e}^{m_{1}} P_{e}^{m_{2}}\left[1-P_{e}^{d_{1}}-P_{e}^{d_{2}}\right]+P_{e}^{m_{1}} P_{e}^{d_{2}}+P_{e}^{m_{2}} P_{e}^{d_{1}} \\
& +P_{e}^{d_{12}}\left[1-P_{e}^{m_{1}}-P_{e}^{m_{2}}+P_{e}^{m_{1}} P_{e}^{m_{2}}\right] \\
& +P_{e}^{m a}\left[P_{e}^{m_{1}}\left(1-P_{e}^{m_{2}}\right)\left(1-P_{e}^{d_{2}}\right)\right] \\
& +P_{e}^{n a}\left[\left(1-P_{e}^{m_{1}}-P_{e}^{m_{2}}+P_{e}^{m_{1}} P_{e}^{m_{2}}\right)\left(P_{e}^{d_{1}}-P_{e}^{d_{12}}\right)\right] \\
\approx & P_{e}^{m_{1}} P_{e}^{m_{2}}+P_{e}^{m_{1}} P_{e}^{d_{2}}+P_{e}^{m_{2}} P_{e}^{d_{1}}+P_{e}^{d_{12}} \\
& +P_{e}^{m a} P_{e}^{m_{1}}+P_{e}^{n a} P_{e}^{d_{1}} .
\end{aligned}
$$


Similarly, the average number of resource usage $N^{I t}$ is obtained as

$$
\begin{aligned}
N^{I t}= & N^{I_{1}}+N_{a n}^{I_{1}}+N^{I_{2 a}}+N^{I_{2 b}} \\
= & m_{1}+d_{1}+P_{e}^{a n}\left(1-P_{e}^{m_{1}}\right)\left(1-P_{e}^{d_{1}}\right)\left(m_{2}+d_{1}\right) \\
& +P_{e}^{d_{1}}\left(1-P_{e}^{m_{1}}\right)\left(1-P_{e}^{n a}\right)\left(m_{2}+d_{1}\right) \\
& +P_{e}^{m_{1}}\left(1-P_{e}^{m a}\right)\left(m_{2}+d_{2}\right) \\
\approx & m_{1}+d_{1}+P_{e}^{m_{1}}\left(m_{2}+d_{2}\right)+\left(P_{e}^{d_{1}}+P_{e}^{a n}\right)\left(m_{2}+d_{1}\right) .
\end{aligned}
$$

As URLLC deal with low values of errors, the relative cross products are negligible that make the applied approximations in (10) and (11) reasonable. In Section V, the accuracy of these approximations are validated.

From (10), we realize that the probability of error in metadata, along with the miss-detection of the feedback signals, are not negligible and have a notable impact on the outage probability. This is unlike most of the literature studies (e.g. [53]-[55], [57]) which assume ideal metadata transmission and mainly focus on data outage probability (i.e., $P_{e}^{d_{12}}$ ). The overhead and the impact of metadata and UL feedback signals on the required resources are visible in (11), where we observe that metadata plays an important role on the network resource utilization. Therefore, it is essential to design and optimize the transmission performance of URLLC by jointly taking into account the data, metadata, and impairments in feedback channel.

We formulate a resource allocation problem to minimize the number of allocated resources while satisfying the quality of service requirements of URLLC. The optimization is expressed as:

$$
\begin{aligned}
& \min _{d_{i}, m_{i}} N^{I t} \\
& \text { S.t. C1I: } P_{\text {out }}^{I t} \leq P_{\text {out }}^{\text {tar }} \\
& \text { C2I: } E\left(m_{i}, \gamma, M\right)=P_{e}^{m_{i}}, \quad i=1,2, \\
& \text { C3I: } E\left(d_{i}, \gamma, D\right)=P_{e}^{d_{i}}, \quad i=1,2, \\
& \text { C4I: } \begin{cases}E\left(d_{1}, 2 \gamma, D\right)=P_{e}^{d_{12}} & \text { for CC-HARQ, } \\
E\left(2 d_{1}, \gamma, D\right)=P_{e}^{d_{12}} & \text { for IR-HARQ, }\end{cases} \\
& \text { C5I: } d_{i}, m_{i} \in \mathbb{N}, \quad i=1,2 .
\end{aligned}
$$

Constraints C1I guarantees the reliability target. Constraints C2I-C4I are the error probabilities corresponding to the channel allocations of data and metadata in the initial transmission and the retransmission. Finally, C5I indicates that the number of allocated resources are positive integers. Note that we have implicitly taken into account the latency requirement by considering that a maximum of one retransmission can be accommodated within the given latency budget [58], [59]. In (12) the objective function and constraint $\mathrm{C} 1 \mathrm{I}$ are non-convex. The equality constraints C2I-C4I are not affine and C5I is integer. Thus, it belongs to the family of integer non-convex optimization problems, difficult to solve with polynomial complexity. In Section IV-A, we present a solution based on consecutive convex optimization to tackle the non-convexity in (12).

\section{B. Joint Encoding of Data and Metadata}

Assuming joint encoding of the metadata and the data to a single codeword of length $n_{1}$, the probability of success in the first attempt $P_{\text {succ }}^{J_{1}}$ is given by

$$
\begin{aligned}
& P_{\text {succ }}^{J_{1}}=1-P_{e}^{J_{1}}, \\
& P_{e}^{J_{1}}=E\left(n_{1}, \gamma, M+D\right) .
\end{aligned}
$$

The UE sends ACK following correct decoding. Since, both metadata and data are encoded (decoded) together, we assume there are no possibilities for sending NACK as well as the HARQ gain of combining data packets after the retransmission. If an ACK is not received, the BS assumes failure and retransmits the packet with a blocklength of $n_{2}$ and BLEP of $P_{e}^{J_{2}}$. Thus, the success probability following the second transmission $P_{\text {succ }}^{J_{2}}$ is given by

$$
P_{\text {succ }}^{J_{2}}=P_{e}^{J_{1}}\left(1-P_{e}^{m a}\right)\left(1-P_{e}^{J_{2}}\right) .
$$

Consequently, for joint encoding of data and metadata, the overall outage probability $P_{\text {out }}^{J t}$ is calculated as

$$
\begin{aligned}
P_{\text {out }}^{J t} & =1-P_{\text {succ }}^{J_{1}}-P_{\text {succ }}^{J_{2}} \\
& =P_{e}^{J_{1}}\left(P_{e}^{m a}+P_{e}^{J_{2}}-P_{e}^{m a} P_{e}^{J_{2}}\right) \approx P_{e}^{J_{1}} P_{e}^{m a}+P_{e}^{J_{1}} P_{e}^{J_{2}}
\end{aligned}
$$

The corresponding average number of allocated resources $N^{J t}$ equals

$$
\begin{aligned}
N^{J t} & =n_{1}+P_{e}^{J_{1}}\left(1-P_{e}^{m a}\right) n_{2}+\left(1-P_{e}^{J_{1}}\right) P_{e}^{a m} n_{2} \\
& \approx n_{1}+P_{e}^{J_{1}} n_{2}+P_{e}^{a m} n_{2},
\end{aligned}
$$

where $P_{e}^{a m}$ is the probability that ACK feedback is not detected correctly by the BS which gives rise to a redundant retransmission.

The resource allocation problem for joint encoding of data and metadata is formulated as follows

$$
\begin{aligned}
& \min _{n_{i}} N^{J t} \\
& \text { S.t.: } \text { C1J: } P_{\text {out }}^{J t}=P_{e}^{J_{1}} P_{e}^{m a}+P_{e}^{J_{1}} P_{e}^{J_{2}} \leq P_{\text {out }}^{\text {tar }}, \\
& \text { C2J: } E\left(n_{i}, \gamma, M+D\right)=P_{e}^{J_{i}}, \quad i=1,2, \\
& \text { C3J: } n_{i} \in \mathbb{N} \quad i=1,2 .
\end{aligned}
$$

Similar to (12), the objective function and constraint $\mathrm{C} 1 \mathrm{~J}$ are non-convex. Constraint $\mathrm{C} 2 \mathrm{~J}$ is not affine, and finally $\mathrm{C} 3 \mathrm{~J}$ is integer. Accordingly, problem (17) is also integer non-convex optimization.

\section{Proposed Low-Complexity NeAR-Optimum SOLUTION}

\section{A. In-Resource Control Signalling}

In order to solve the optimization problem (12), an efficient near-optimum low-complexity solution is proposed. In the rest of the paper, we assume CC-HARQ. However, similar approach can be applied to IR-HARQ. We handle the nonconvexity of the problem by developing an algorithm based on successive solving a convex optimization problem through the following steps. 
- Step-1: Integer relaxation. First, we relax the constraint C5I in (12) and assume channel uses can be positive real numbers (i.e. $d_{i}, m_{i} \in \mathbb{R}^{+}$).

- Step-2: Convert non-convex functions to convex form. The objective function and constraint C1I in (12) are non-convex posynomial functions [80]. To handle the non-convexity, we introduce new variables as: $x_{1} \triangleq$ $\ln m_{1}, x_{2} \triangleq \ln d_{1}, x_{3} \triangleq \ln m_{2}, x_{4} \triangleq \ln d_{2}, y_{1} \triangleq$ $\ln P_{e}^{m_{1}}, y_{2} \triangleq \ln P_{e}^{d_{1}}, y_{3} \triangleq \ln P_{e}^{m_{1}}, y_{4} \triangleq \ln P_{e}^{m_{2}} y_{5} \triangleq$ $\ln P_{e}^{d_{12}}$. Revising problem (12) with respect to new variables and substituting the objective function and constraint C1I with their logarithmic form result in

$$
\begin{aligned}
\min _{x_{i}, y_{i}} \ln \left[e^{x_{1}}+e^{x_{2}}+e^{y_{1}+x_{3}}+e^{y_{1}+x_{4}}+e^{y_{2}+x_{2}}\right. & \\
& \left.+e^{y_{2}+x_{3}}+P_{e}^{a n}\left(e^{x_{2}}+e^{x_{3}}\right)\right],
\end{aligned}
$$

S.t. C1a: $\ln \left[e^{y_{1}+y_{3}}+e^{y_{1}+y_{4}}+e^{y_{2}+y_{3}}\right.$

$$
\left.+e^{y_{5}}+P_{e}^{m a} e^{y_{1}}+P_{e}^{n a} e^{y_{2}}\right]-\ln P_{\text {out }}^{\text {tar }} \leq 0,
$$

C2Ia: $E\left(e^{x_{i}}, \gamma, M\right)=e^{y_{i}}, \quad i=1,3$,

C3Ia: $E\left(e^{x_{i}}, \gamma, D\right)=e^{y_{i}}, \quad i=2,4$,

C4Ia: $E\left(e^{x_{1}}, 2 \gamma, D\right)=e^{y_{5}}$,

C5Ia: $x_{i} \in \mathbb{R}^{+}, \quad i \in\{1, \ldots, 4\}$,

C6Ia: $y_{i} \in \mathbb{R}, \quad i \in\{1, \ldots, 5\}$.

In the revised optimization problem (18), the objective function and constraint C1Ia are standard convex form [80].

- Step-3: Modify equality constraints. Constraints C2IaC4Ia are non-affine equality constraints. Without loss of optimality, we modify them to inequality constraints as

C2Ib: $E\left(e^{x_{i}}, \gamma, M\right)-e^{y_{i}} \leq 0, \quad i=1,3$,

C3Ib: $E\left(e^{x_{i}}, \gamma, D\right)-e^{y_{i}} \leq 0, \quad i=2,4$,

C4Ib: $E\left(e^{x_{2}}, 2 \gamma, D\right)-e^{y_{5}} \leq 0$.

Proof. Please refer to Appendix A.

- Step-4: Difference of convex functions. Constraints C2Ib-C4Ib are the difference of two convex functions.

Proof. Please refer to Appendix B.

To handle the non-convexity of constraints $\mathrm{C} 2 \mathrm{Ib}-\mathrm{C} 4 \mathrm{Ib}$, we employ successive convex approximation [48], [81]. Applying Taylor expansion for the convex functions, the first order approximation of $e^{y_{i}}$ with respect to an initial point $y_{i}^{0}$ is expressed as

$$
e^{y_{i}^{0}}+e^{y_{i}^{0}}\left(y_{i}-y_{i}^{0}\right) \leq e^{y_{i}}
$$

which is an affine function of $y_{i}$. Substituting (20) into
(19), we obtain the following problem

$$
\begin{array}{r}
\min _{x_{i}, y_{i}} \ln \left[e^{x_{1}}+e^{x_{2}}+e^{y_{1}+x_{3}}+e^{y_{1}+x_{4}}+e^{y_{2}+x_{2}}\right. \\
\left.+e^{y_{2}+x_{3}}+P_{e}^{a n}\left(e^{x_{2}}+e^{x_{3}}\right)\right],
\end{array}
$$

S.t. C1Ia: $\ln \left[e^{y_{1}+y_{3}}+e^{y_{1}+y_{4}}+e^{y_{2}+y_{3}}\right.$

$$
\left.+e^{y_{5}}+P_{e}^{m a} e^{y_{1}}+P_{e}^{n a} e^{y_{2}}\right]-\ln P_{\text {out }}^{\text {tar }} \leq 0,
$$

C2Ic: $E\left(e^{x_{i}}, \gamma, M\right)-e^{y_{i}^{0}}\left(1+y_{i}-y_{i}^{0}\right) \leq 0, i=1,3$,

C3Ic: $E\left(e^{x_{i}}, \gamma, D\right)-e^{y_{i}^{0}}\left(1+y_{i}-y_{i}^{0}\right) \leq 0, i=2,4$,

C4Ic: $E\left(e^{x_{2}}, 2 \gamma, D\right)-e^{y_{5}^{0}}\left(1+y_{5}-y_{5}^{0}\right) \leq 0$,

C5Ic: $x_{i} \in \mathbb{R}^{+} \quad i \in\{1, \ldots, 4\}$,

C6Ic: $y_{i} \in \mathbb{R} \quad i \in\{1, \ldots, 5\}$.

- Step-5: Iterative solving of standard optimization problem. In (21), the objective and constraints are convex functions forming a standard convex optimization problem that can be solved via optimization toolbox with polynomial time. Based on these analyses, we apply an iterative algorithm to find a sub-optimal solution for problem (12). The convex optimization problem in (21) is solved with the initial points $y_{i}^{0}$. The initial points are then updated with the optimal solutions of the previous iteration. Algorithm 1 summarizes the steps of the proposed solution.

Algorithm 1 Proposed Solution for the Optimization Problem (12)

1: Initialize: The initial points $e^{y_{i}^{0}}$, the iteration number $k=$ 0 , and the maximum number of iterations $K_{\max }$.

2: Repeat:

3: Solve the convex optimization problem (21) with $e^{y_{i}^{k}}$.

4: $\quad$ Set $k=k+1$.

5: Update $e^{y_{i}^{k}}=e^{y_{i}}$.

6: Until: $k=K_{\max }$.

: Return: $e^{x_{i}}$.

\section{B. Joint Encoding of Data and Metadata}

Problem (17) has similar structure as that of (12). Thereby, applying the same approaches as in Section IV-A yields a standard convex optimization

$$
\begin{aligned}
\min _{w_{i}, z_{i}} \ln \left[e^{w_{1}}+e^{z_{1}+w_{2}}+P_{e}^{a m} e^{w_{2}}\right], \\
\text { S.t.: C1Ja: } \ln \left[e^{z_{1}} P_{e}^{m a}+e^{z_{1}+z_{2}}\right]-\ln P_{\text {out }}^{\text {tar }} \leq 0, \\
\text { C2Ja: } E\left(e^{w_{i}}, \gamma, D+M\right)-e^{z_{i}^{0}}\left(1+z_{i}-z_{i}^{0}\right) \leq 0, \\
\text { C3Ja: } w_{i} \in \mathbb{R}^{+}, \quad i=1,2, \\
\text { C4Ja: } z_{i} \in \mathbb{R}, \quad i=1,2,
\end{aligned}
$$

where $w_{i} \triangleq \ln n_{i}, z_{i} \triangleq \ln P_{e}^{J_{i}}$. Algorithm 2 presents the steps toward solving problem (17).

By solving optimization problems (12) and (17), we derive the minimum average number of required resources while satisfying URLLC targets. Note that imposing additional constraints in terms of available resources may likely lead to a 
sub-optimal result (i.e. higher number of required resources) or may not satisfy the reliability requirement.

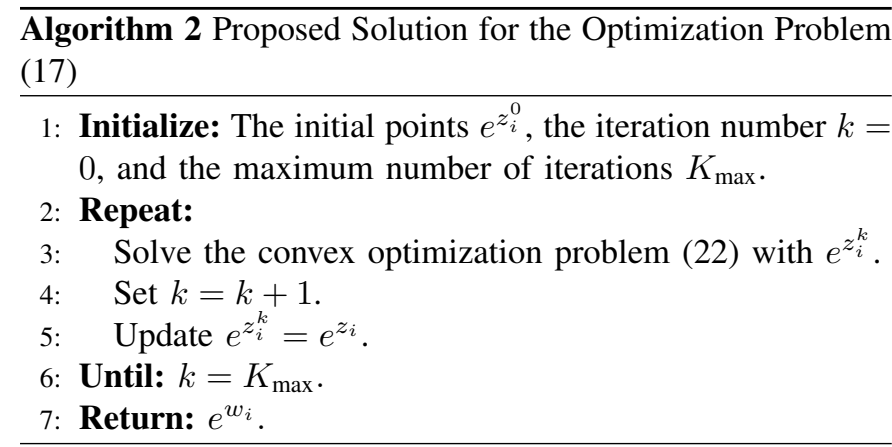

\section{NUMERICAL RESULTS}

Numerical results evaluating the performance of different multiplexing schemes are presented in the following. For simulation parameters, we assume equal false-alarm probability $P_{e}^{f a}$ for $P_{e}^{n a}$ and $P_{e}^{m a}$ (i.e. $P_{e}^{n a}=P_{e}^{m a}=P_{e}^{f a}=10^{-5}$ ). Also, to cover the asymmetric detection of feedback signals in URLLC we assume $P_{e}^{a n}=P_{e}^{a m}=5 P_{e}^{f a}=5 \times 10^{-5}$. We set the number of maximum iterations $K_{\max }=5$ for Algorithms 1 and 2 .

\section{A. In-Resource Control Signalling}

Fig. 1 depicts the resource usage performance gains of sending URLLC payloads, assuming in-resource control channel multiplexing with respect to outage probability targets ranging from $P_{\text {out }}^{\text {tar }}=10^{-4}$ to $P_{\text {out }}^{\text {tar }}=10^{-7}$ and for different channel conditions. We assume data and metadata size are $D=32$ and $M=16$ bytes, respectively [61], [77]. The gains are compared against the baseline scenario with a single transmission in which both data metadata are encoded with the same BLEP equal $P_{e}^{d_{1}}=P_{e}^{m_{1}}=\frac{1}{2} P_{\text {out }}^{\text {tar }}$. Considering cases with two transmissions, we present results for three scheduling schemes: i) Transmission with constant BLEP (i.e. $\left.P_{e}^{d_{i}}=P_{e}^{m_{i}}=P_{e}, i=1,2\right)$. ii) The proposed solution provided by Algorithm 1. iii) The optimal solution obtained by performing an exhaustive search over the feasible set of points and without approximations in (10) and (11).

Assuming two transmissions with equal BLEP $P_{e}$ for both metadata and data, the expression of total outage probability in equation (10) is further simplified to

$$
P_{\text {out }}^{S t}=3 P_{e}{ }^{2}+P_{e}\left(P_{e}^{n a}+P_{e}^{m a}\right)+P_{e}^{12} \leq P_{\text {out }}^{\text {tar }} .
$$

Solving (23) with equality constraint to minimize the number of resources, results

$$
\begin{aligned}
P_{e}^{d_{i}}=P_{e}^{m_{i}} & =P_{e} \approx-\frac{\left(P_{e}^{n a}+P_{e}^{m a}\right)}{6} \\
& +\frac{\left(\left(P_{e}^{n a}+P_{e}^{m a}\right)^{2}+12 P_{\text {out }}^{\text {tar }}\right)^{\frac{1}{2}}}{6}, \quad i=1,2 .
\end{aligned}
$$

Accordingly, the number of resources can be calculated using (3). Fig. 1 reveals significant resource efficiency enhancements with two transmissions. As we observe, the gain increases

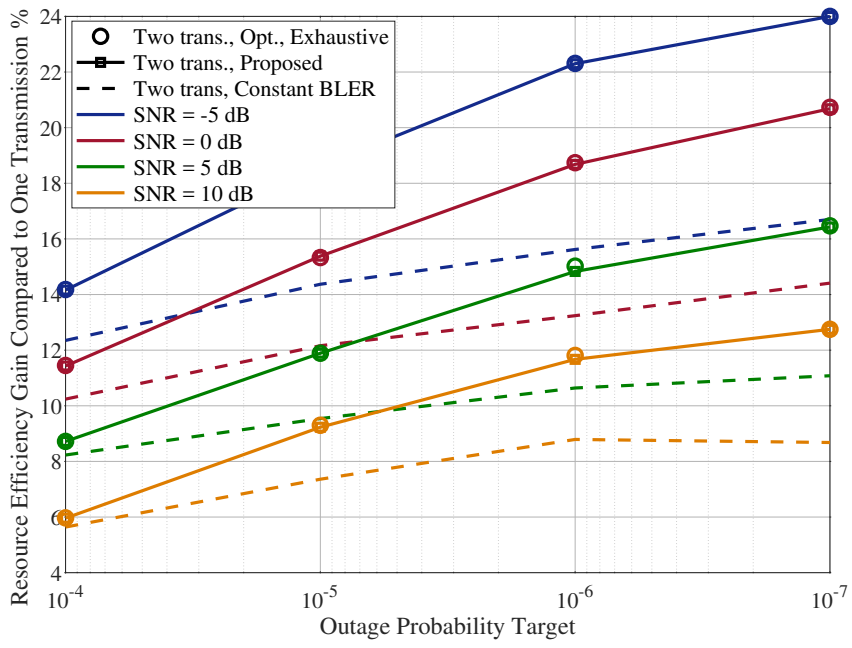

Fig. 1: Performance analysis of in-resource control signalling for different outage probabilities and channel conditions with $M=16$ and $D=32$ bytes. $P_{e}^{n a}=P_{e}^{m a}=10^{-5}$ and $P_{e}^{a n}=$ $P_{e}^{a m}=5 \times 10^{-5}$.

with tighter reliability requirements. For instance, at the outage of $P_{\text {out }}^{\text {tar }}=10^{-7}(99.99999 \%$ reliability $)$ and $-5 \mathrm{~dB}$ SNR, constants BLEP is $16 \%$ more resource-efficient as compared to single-shot scheduling. Moreover, the proposed variable error-rate scheduling provides better performance and achieves $24 \%$ gain in resource usage. This is because the proposed algorithm schedules the initial transmission with higher rates to minimize the resources. Failure to decode metadata at the first transmission, both data and metadata are scheduled with lower coding-rate to further improve the reliability. On the other hand, if the UE could not decode the data, HARQ retransmission is scheduled with robust metadata, and the data quality is enhanced by HARQ combining of the received data packets.

Comparing the results from the proposed algorithm with those of optimal solutions by performing exhaustive search operation, we observe that our algorithm performs well and approaches a similar performance close to the optimal point solution. As the exhaustive search solution is driven by investigating the original resource allocation problem (without approximations), it confirms that the applied approximations in (10) and (11) are accurate and valid. It is also interesting to note that since a fixed power spectrum density is applied over the assigned bandwidth, the provided gain is two dimensional, given that it reduces the assigned spectrum and the power with the same amount.

Fig. 2 shows the resource efficiency gain for different metadata and data set sizes assuming SNR of $\gamma=0 \mathrm{~dB}$. For each set, the achieved gain is calculated by comparing against the results of single transmission with the same metadata data sizes. We observe that the gain is higher for short payload sizes. Assuming $M=10$ and $D=16$ bytes, the proposed solution is $27 \%$ more resource efficient at $10^{-7}$ outage probability. Increasing the data size to $D=50$ bytes, the gain reduces to $18 \%$. This is due to the fact that the 


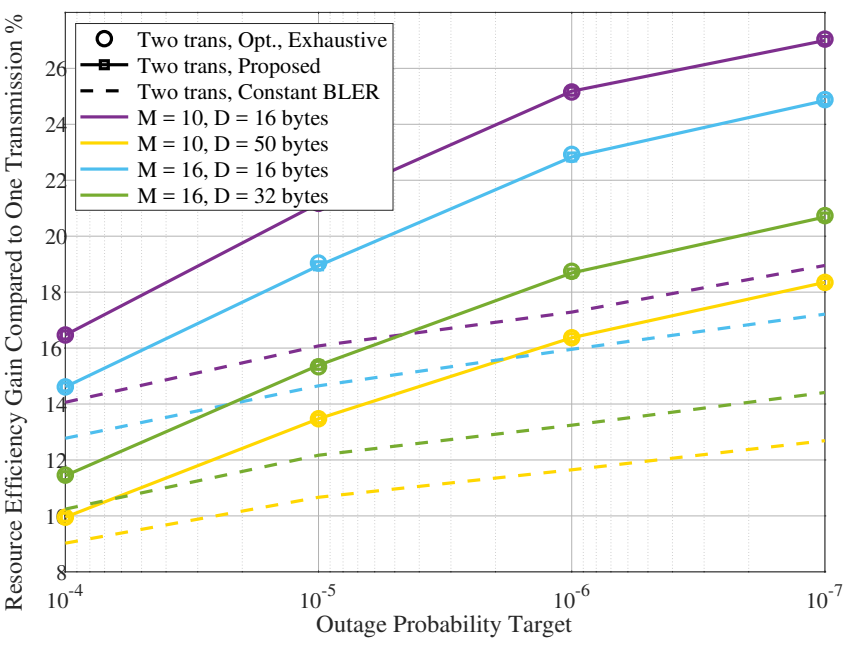

Fig. 2: Performance analysis of in-resource control signalling for different metadata and data sizes with $\gamma=0 \mathrm{~dB} . P_{e}^{n a}=$ $P_{e}^{m a}=10^{-5}$ and $P_{e}^{a n}=P_{e}^{a m}=5 \times 10^{-5}$.

impairment of short packet transmission decreases as the blocklength grows and the achievable rate converges to the Shannon Capacity. This in turn facilitates low-error scheduling with single transmission.

\section{B. Joint Encoding of Data and Metadata}

In Figs. 3 and 4 we evaluate the performance of joint encoding of data and metadata. Fig. 3 plots the achieved gain versus the outage probability for different transmission schemes and SNR values. For the baseline single transmission, the minimum number of required resources $n_{1}$ is calculated based on (3) such that $E\left(n_{1}, \gamma, M+D\right)=P_{\text {out }}^{\text {tar }}$. For equal constant BLEP with retransmission, the error probabilities are obtained from (15) as

$$
P_{e}^{J_{1}}=P_{e}^{J_{2}} \approx \frac{-P_{e}^{m a}}{2}+\frac{\left(\left(P_{e}^{m a}\right)^{2}+4 P_{\text {out }}^{\text {tar }}\right)^{\frac{1}{2}}}{2} .
$$

As can be observed, the proposed solution improves the performance by reducing the number of resources required to guarantee the desired reliability targets. At SNR of $\gamma=0$ $\mathrm{dB}$ and for $99.99999 \%$ reliability, it provides $16 \%$ gain in comparison to the baseline single transmission. Moreover, the performance is very close to that of the optimal exhaustive search solution. The results in Figs. 1 and 3 show that retransmission is more favourable at low-SNR regimes. The achieved gain of retransmission decreases with an increase in channel quality. The reason is that reliability significantly enhances at high SNRs. Therefore, it is also possible to achieve the reliability target with a relatively low number of resources in a single allocation.

Fig. 4 shows the performance for different metadata and data sizes. Similar to the results of Fig. 2, we observe retransmission is more beneficial for small payloads and the gain decreases for larger blocklengths. Comparing in-resource control signalling with joint encoding, we observe that the relative improvements of enabling retransmission are higher

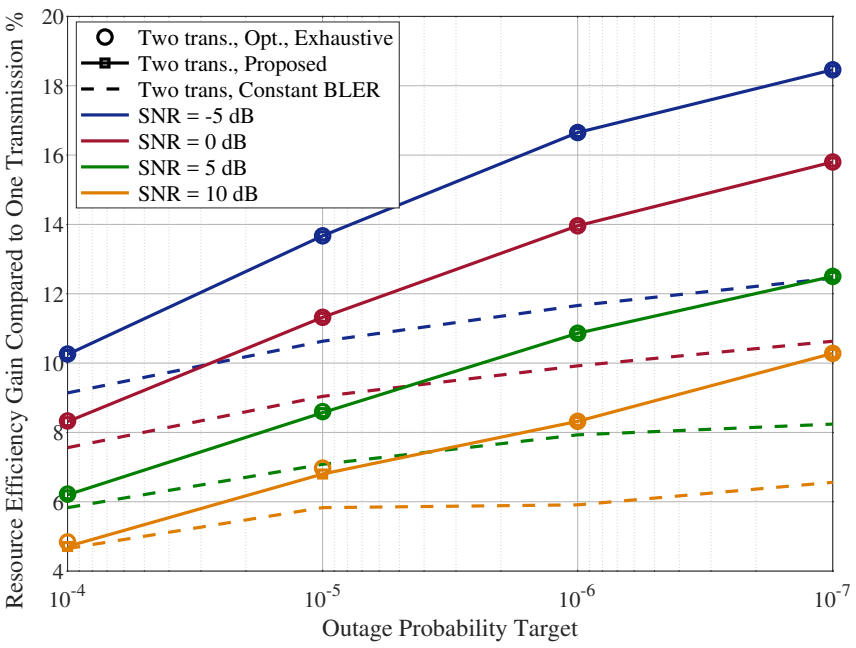

Fig. 3: Performance analysis of joint encoding of data and metadata for different outage probabilities and channel conditions with $M=16$ and $D=32$ bytes. $P_{e}^{m a}=10^{-5}$ and $P_{e}^{a m}=5 \times 10^{-5}$.

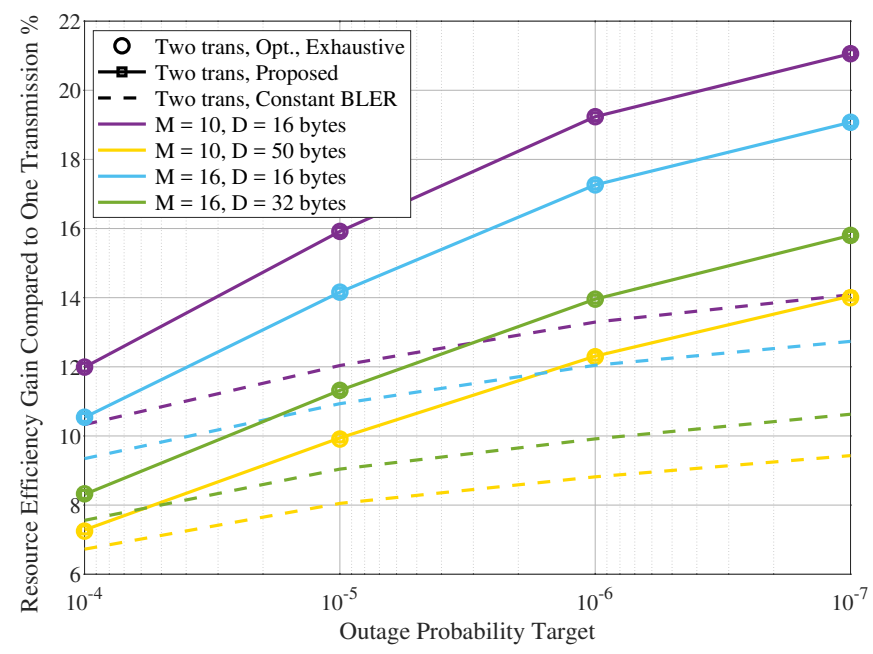

Fig. 4: Performance analysis of joint encoding for different metadata and data sizes with $\gamma=0 \mathrm{~dB} . P_{e}^{n a}=P_{e}^{m a}=10^{-5}$ and $P_{e}^{a n}=P_{e}^{a m}=5 \times 10^{-5}$.

for in-resource control transmission. Assuming $M=D=16$ bytes and at the outage probability of $10^{-6}$, the proposed algorithm results in $23 \%$ gain as compared to baseline case for in-resource control signalling. While for joint encoding, $17 \%$ improvement is achieved. This is because of the capability of the joint transmission scheme to encode both the metadata and data with a larger codeword that diminishes short blocklength inefficiency.

\section{Performance Comparison}

In Fig. 5, we provide a comparison between in-resource control scheduling and joint encoding of data and metadata. The relationship between resource efficiency and feedback errors is also further investigated. The figure plots the minimum number of required resource elements versus operating SNRs 
TABLE II: URLLC BLEP targets for different multiplexing schemes and outage reliabilities. $M=10$ and $D=50$ bytes, $P_{e}^{n a}=P_{e}^{m a}=10^{-5}, P_{e}^{a n}=P_{e}^{a m}=5 \times 10^{-5}, \gamma=0 \mathrm{~dB}$.

\begin{tabular}{|c|c|c|c|c|c|c|c|}
\hline \multirow{2}{*}{\multicolumn{2}{|c|}{ Scenario }} & \multicolumn{3}{|c|}{$P_{\text {out }}^{t a r}=10^{-5}$} & \multicolumn{3}{|c|}{$P^{\operatorname{tar}}=10^{-7}$} \\
\hline & & \multirow{2}{*}{$\begin{array}{c}\text { First Transmission } \\
2 \times 10^{-2}\end{array}$} & \multicolumn{2}{|c|}{ Second Transmission } & First Transmission & \multicolumn{2}{|c|}{ Second Transmission } \\
\hline \multicolumn{2}{|c|}{ Joint Encoding } & & \multirow{2}{*}{\multicolumn{2}{|c|}{$\frac{4 \times 10^{-4}}{2 \times 10^{-4}}$}} & $1 \times 10^{-2}$ & \multirow{2}{*}{\multicolumn{2}{|c|}{$\frac{2 \times 10^{-7}}{1 \times 10^{-7}}$}} \\
\hline \multirow{2}{*}{$\begin{array}{l}\text { In-resource Control } \\
\text { Signalling }\end{array}$} & Metadata & $8 \times 10^{-3}$ & & & $3 \times 10^{-3}$ & & \\
\hline & Data & $2 \times 10^{-2}$ & $\begin{array}{c}\text { NACK } \\
2 \times 10^{-2}\end{array}$ & $\begin{array}{c}\text { DTX } \\
4 \times 10^{-4}\end{array}$ & $6 \times 10^{-3}$ & $\begin{array}{c}\text { NACK } \\
6 \times 10^{-3}\end{array}$ & $\begin{array}{c}\text { DTX } \\
1.5 \times 10^{-7}\end{array}$ \\
\hline
\end{tabular}

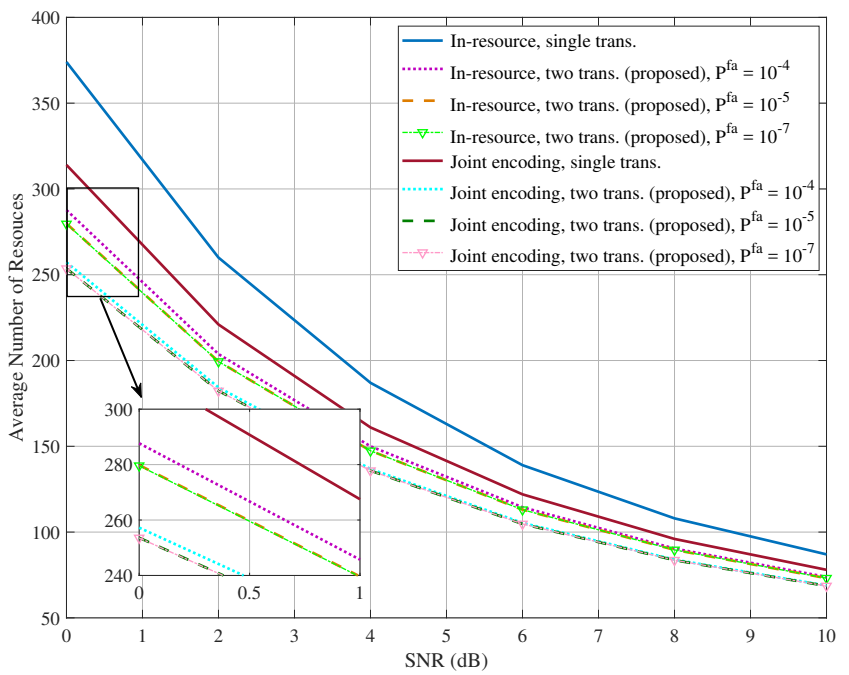

Fig. 5: Performance comparison of in-resource control signalling and joint encoding of data and metadata with different feedback errors, assuming $P_{\text {out }}^{\text {tar }}=10^{-6}, M=10$ and $D=16$ bytes. $P_{e}^{n a}=P_{e}^{m a}=P_{e}^{f a}, P_{e}^{a n}=P_{e}^{a m}=5 P_{e}^{f a}$.

for different scheduling schemes. Benefiting from sending information over a larger codeword and in comparison to in-resource control signalling, joint encoding shows superior resource efficiency for both single and two-transmission schemes. Recall that, though joint encoding is more efficient, its relative gain with respect to single transmission is lower than in-resource control signalling, as highlighted by the previous results. Assuming single transmission and at $5 \mathrm{~dB} \mathrm{SNR}$, it provides $14 \%$ gain over the in-resource control transmission. With two transmissions and by applying the proposed optimization, the difference decreases to $8 \%$. However, the gain comes at the expense of higher complexity (more required energy and processing time) at the receiver. Detailed comparison between in-resource control signalling and joint encoding in terms of complexity and energy consumption depends on several practical parameters such as coding scheme, coding rate, code length, decoding method, number of codebooks, size of searching area [82], [83]. Such aspects are left for future studies. Moreover, the plot shows that the performance difference decreases with improving channel conditions. Additionally, we observe that feedback errors have lower (in comparison to data and metadata) impact on the resource usage. Reducing feedback error from $10^{-4}$ to $10^{-7}$ results around $1 \%$ improvement of the resource efficiency at $5 \mathrm{~dB}$ SNR.
Finally, Table II presents BLEP targets of the first and the second transmissions for different multiplexing schemes and outage probability. The results illustrate that the BLEP targets in the first transmission are orders of magnitude higher than the second ones for both in-resource control signalling and joint encoding approaches. This is beneficial to enhance the throughput within the initial allocation. However, the error probability increases with the coding rates that leads additional resource assignment for retransmission. Therefore, the provided gain is bounded by the failure rate, outage probability target, and the error probability in feedback channel (that limits the applicability of retransmission).

By comparing the results of joint encoding at $10^{-5}$ and $10^{-7}$ outage probability levels, we find similar BLEPs for the initial transmissions. But the BLEP targets significantly vary for the second retransmissions. This highlights the benefits of enabling variable rate retransmission to well accommodate with different reliability targets. A similar trend is observed considering in-resource control signalling. In this scheme, based on the source of retransmission, we notice that different BLEP targets are needed to be set for data scheduling. Due to the potential gain achieved from CC-HARQ, retransmitting the same data packet as the original one guarantees the reliability requirements if NACK is received. In the case of DTX, transmission with a more robust MCS is desirable.

The provided algorithm can be easily realized for practical implementations by generating offline such above tables that take those input parameters and return either the desired BLER target of the data or the aggregation level of the metadata as output. Hence, for each new data transmission, the BS simply performs a look-up in the corresponding table and selects the aggregation level and MCS for the metadata and data, respectively, to achieve the most resource-efficient transmission, while still fulfilling the QoS requirements for the users. This relaxes the need for solving the optimization problem each time a new packet arrives and therefore does not affect processing time requirement of URLLC services.

The proposed solution can be directly applied in multi-cell multi-user or mixed URLLC and eMBB scenarios to enhance the network performance. In such cases and for each UE, the BS determines the best link adaptation strategy that minimizes the number of required resources. The link adaptation results (i.e. BLER targets) are then directly used as an input for multiplexing problems [10], [62]. Scheduling URLLC traffic with lower number of resources is advantageous as the network can schedule more URLLC payloads to reduce the queuing delay or serve a different traffic type (e.g. eMBB) over the available resources. 


$$
\begin{aligned}
\frac{\partial^{2} E\left(e^{x}, \gamma, b\right)}{\partial^{2} x} & =\frac{e^{\frac{-1}{2}\left(\frac{e^{x} C(\gamma)-b}{\sqrt{e^{x} V(\gamma)}}\right)^{2}}}{4 \sqrt{2 \pi}}\left[\frac{C^{3}(\gamma) e^{3 x}-b^{3}-b^{2} C(\gamma) e^{x}+b C^{2}(\gamma) e^{2 x}+3 b V(\gamma) e^{x}+C(\gamma) V(\gamma) e^{2 x}}{e^{x} V(\gamma) \sqrt{e^{x} V(\gamma)}}-\frac{e^{x} C(\gamma)+b}{\sqrt{e^{x} V(\gamma)}}\right] \\
& =\frac{e^{\frac{-1}{2}\left(\frac{e^{x} C(\gamma)-b}{\sqrt{e^{x} V(\gamma)}}\right)^{2}}}{4 e^{x} V(\gamma) \sqrt{2 \pi e^{x} V(\gamma)}}\left(e^{x} C(\gamma)-b\right)\left[C^{2}(\gamma) e^{2 x}+2 C(\gamma) e^{x} b+b^{2}-V(\gamma) e^{x}\right] \\
& =\frac{e^{\frac{-1}{2}\left(\frac{e^{x} C(\gamma)-b}{\sqrt{e^{x} V(\gamma)}}\right)^{2}}}{4 e^{x} V(\gamma) \sqrt{2 \pi e^{x} V(\gamma)}} \underbrace{\left(e^{x} C(\gamma)-b\right)}_{=\Pi} \underbrace{\left(C(\gamma) e^{x}+b-\sqrt{V(\gamma) e^{x}}\right)}_{=\Phi}\left(C(\gamma) e^{x}+b+\sqrt{V(\gamma) e^{x}}\right) .
\end{aligned}
$$

\section{CONCLUSION}

This paper studied downlink radio resource allocation for URLLC in 5G NR. Two multiplexing methodologies namely as in-resource control signalling and joint encoding (decoding) of data and metadata were investigated. We proposed an analytical framework to evaluate the allocated resources and the outage probability of URLLC scheduling. It was shown that non-ideal transmission of control information has a significant impact on the system overhead and reliability of URLLC. For each scheme, we formulated an optimization problem comprising joint link adaptation for data and metadata to minimize resource allocation while guaranteeing URLLC requirements. Since the problems are integer nonconvex optimizations, solutions based on successive convex optimizations were proposed. Numerical analyses showed that the proposed algorithms perform close to the optimal solutions, significantly reduce the resource usage, and achieve up to $27 \%$ resource utilization improvement. Future studies could examine the impact of imperfect (delayed) channel knowledge at transceivers and analyse the performance of multi-user multiplexing.

\section{APPENDIX A}

We show that constraints C2Ia-C4Ia are hold with equality at the optimum point. To this end, we first prove that the decoding error probability is always a decreasing function with respect to $x$. The partial derivative of the function $E\left(e^{x}, \gamma, b\right)$ with respect to $x$ is given by

$$
\frac{\partial E\left(e^{x}, \gamma, b\right)}{\partial x}=-\frac{e^{x} C(\gamma)+b}{2 \sqrt{2 \pi e^{x} V(\gamma)}} e^{\frac{-1}{2}\left(\frac{e^{x} C(\gamma)-b}{\sqrt{e^{x} V(\gamma)}}\right)^{2}} \leq 0
$$

meaning that $E(\cdot)$ is monotonically decreasing function of $x$.

Applying the contradiction theory, let us assume $\left\{x_{i}^{\star}, y_{i}^{\star}\right\}$ are the optimal solutions of (18) satisfying at least one of the constraints with non-equality (i.e. $\left.E\left(e^{x_{j}^{\star}}, \gamma, b\right)<e^{y_{j}^{\star}}\right)$. In this case, the achieved minimum number of channel uses is denoted by $N^{\star}$. We denote $x_{j}^{\star \star}$ as the solution of $E\left(e^{x_{j}^{\star \star}}, \gamma, b\right)=$ $e^{y_{j}^{\star}}$ can be obtained using (3). Since $E(\cdot)$ is always decreasing with respect to $x$, we have $x_{j}^{\star \star}<x_{j}^{\star}$. Suppose a set of points as $\left\{x_{i, i \neq j}^{\star}, x_{j}^{\star \star}, y_{i}^{\star}\right\}$ resulting $N^{\star \star}$ channel uses. This leads to $N^{\star \star}<N^{\star}$ which is in contradiction with the optimality assumption of $N^{\star}$. Following similar proofs for other cases, we conclude that modifying equality constraints to inequality does not change the optimal solution.

\section{APPENDIX B}

To prove the convexity of decoding error probability, it is sufficient to show that the second derivative of $E\left(e^{x}, \gamma, b\right)$ presented in (26) is positive. In (26), positivity of the term $\Pi$ holds when $e^{x} C(\gamma) \geq b$ implying that $0<E\left(e^{x}, \gamma, b\right) \leq \frac{1}{2}$ which is a valid assumption for URLLC. Also term $\Phi$ has quadratic form and is convex with respect to $e^{\frac{x}{2}}\left(\frac{\partial^{2} \Phi}{\partial^{2} e^{\frac{x}{2}}}=\right.$ $2 C(\gamma) \geq 0)$. Therefore, $\Phi$ is minimized setting $\frac{\partial \Phi}{\partial e^{\frac{x}{2}}}=0$, that results in $e^{\frac{x}{2}}=\sqrt{V(\gamma)} / 2 C(\gamma)$. The minimum value of $\Phi$ is given by

$$
\Phi^{\min }=b-\frac{V(\gamma)}{4 C(\gamma)}
$$

Taking the first derivative of $\Phi^{\text {min }}$ with respect to $\gamma$, we have

$$
\frac{\partial \Phi^{\min }}{\partial \gamma}=\frac{1}{4 \ln 2} \frac{\gamma^{2}+2(\gamma-\ln (1+\gamma))}{(1+\gamma)^{3} \ln ^{2}(1+\gamma)} \text {. }
$$

Given that $\gamma \geq \ln (1+\gamma)$ we conclude $\frac{\partial \Phi^{\min }}{\partial \gamma} \geq 0$, indicating that $\Phi^{\mathrm{min}}$ is monotonically increasing function of $\gamma$. As $\Phi^{\mathrm{min}}$ is also increasing with $b$, it is sufficient to show that $\Phi^{\text {min }} \geq 0$ for few number of information bits and low-value of SNR. Assuming $b=1$ bit and $\gamma=-100 \mathrm{~dB}$, we have $\Phi^{\text {min }}=0.27$. We therefore conclude that $E\left(e^{x}, \gamma, b\right)$ is convex function of $x$. It is straightforward to show that $e^{y}$ is also a convex function with respect to $y$. This completes the proof.

\section{REFERENCES}

[1] 3GPP Technical Specification 38.300, "NR and NG-RAN overall description; stage-2," Version 15.5.0, March 2019.

[2] IMT Vision, "Framework and overall objectives of the future development of IMT for 2020 and beyond," International Telecommunication Union (ITU), Document, Radiocommunication Study Groups, February 2015.

[3] A. Ghosh, A. Maeder, M. Baker, and D. Chandramouli, "5G evolution: A view on 5G cellular technology beyond 3GPP Release 15," IEEE Access, vol. 7, pp. 127639-127 651, 2019.

[4] H. Ji et al., "Ultra-reliable and low-latency communications in 5G downlink: Physical layer aspects," IEEE Wireless Communications, vol. 25, no. 3, pp. 124-130, June 2018.

[5] J. Sachs et al., "5G radio network design for ultra-reliable low-latency communication," IEEE Network, vol. 32, no. 2, pp. 24-31, March 2018.

[6] B. Chang et al., "Optimizing resource allocation in URLLC for real-time wireless control systems," IEEE Transactions on Vehicular Technology, vol. 68, no. 9, pp. 8916-8927, September. 2019.

[7] 3GPP Technical Report 38.913, "Study on scenarios and requirements for next generation access technologies," Version 14.1.0, March 2017.

[8] N. H. Mahmood et al., "Radio resource management techniques for eMBB and mMTC services in 5G dense small cell scenarios," in 2016 IEEE 84th Vehicular Technology Conference (VTC-Fall), Montreal, Canada, September 2016, pp. 1-5. 
[9] K. I. Pedersen et al., "A flexible 5G frame structure design for frequencydivision duplex cases," IEEE Communications Magazine, vol. 54, no. 3, pp. 53-59, March 2016

[10] A. Karimi et al., "Efficient low-complexity packet scheduling algorithm for mixed URLLC and eMBB traffic in 5G," in 2019 IEEE 89th Vehicular Technology Conference (VTC2019-Spring), April 2019, pp. $1-6$.

[11] R. Abreu et al., "System level analysis of eMBB and Grant-Free URLLC multiplexing in uplink," in 2019 IEEE 89th Vehicular Technology Conference (VTC2019-Spring), April 2019, pp. 1-5.

[12] A. Anand, G. D. Veciana, and S. Shakkottai, "Joint scheduling of URLLC and eMBB traffic in 5G wireless networks," in IEEE INFOCOM 2018 - IEEE Conference on Computer Communications, April 2018, pp. 1970-1978.

[13] A. A. Esswie and K. I. Pedersen, "Opportunistic spatial preemptive scheduling for URLLC and eMBB coexistence in multi-user 5G networks," IEEE Access, vol. 6, pp. 38 451-38 463, 2018.

[14] H. Chien, Y. Lin, C. Lai, and C. Wang, "End-to-end slicing with optimized communication and computing resource allocation in multi-tenant 5G systems," IEEE Transactions on Vehicular Technology, vol. 69, no. 2, pp. 2079-2091, February 2020.

[15] L. Feng et al., "Dynamic resource allocation with RAN slicing and scheduling for URLLC and eMBB hybrid services," IEEE Access, vol. 8 , pp. 34 538-34 551, 2020.

[16] A. Anand, G. de Veciana, and S. Shakkottai, "Joint scheduling of URLLC and eMBB traffic in 5G wireless networks," IEEE/ACM Transactions on Networking, pp. 1-14, 2020.

[17] A. Karimi et al., "5G centralized multi-cell scheduling for URLLC: Algorithms and system-level performance," IEEE Access, vol. 6, pp. 72 253-72 262, 2018.

[18] A. Karimi, K. I. Pedersen, and P. Mogensen, "5G URLLC performance analysis of dynamic-point selection multi-user resource allocation," in 2019 16th International Symposium on Wireless Communication Systems (ISWCS), 2019, pp. 379-383.

[19] N. H. Mahmood et al., "Reliability oriented dual connectivity for URLLC services in 5G New Radio," in 2018 15th International Symposium on Wireless Communication Systems (ISWCS), August 2018, pp. $1-6$.

[20] M. Centenaro et al., "System-level study of data duplication enhancements for 5G downlink URLLC," IEEE Access, vol. 8, pp. 565-578, 2020.

[21] G. Berardinelli et al., "Reliability analysis of uplink grant-free transmission over shared resources," IEEE Access, vol. 6, pp. 23 602-23 611, 2018.

[22] Z. Ding, R. Schober, P. Fan, and H. V. Poor, "Simple semi-grantfree transmission strategies assisted by non-orthogonal multiple access," IEEE Transactions on Communications, pp. 1-1, 2019.

[23] T. H. Jacobsen et al., "Multi-cell reception for uplink grant-free ultrareliable low-latency communications," IEEE Access, vol. 7, pp. 80208 80218, 2019.

[24] C. Guo, L. Liang, and G. Y. Li, "Resource allocation for low-latency vehicular communications: An effective capacity perspective," IEEE Journal on Selected Areas in Communications, vol. 37, no. 4, pp. 905917, April 2019

[25] C. F. Liu and M. Bennis, "Ultra-reliable and low-latency vehicular transmission: An extreme value theory approach," IEEE Communications Letters, vol. 22, no. 6, pp. 1292-1295, June 2018.

[26] C. She et al., "Ultra-reliable and low-latency communications in unmanned aerial vehicle communication systems," IEEE Transactions on Communications, pp. 1-1, 2019.

[27] L. Feng et al., "Joint computation offloading and URLLC resource allocation for collaborative MEC assisted cellular-V2X networks," IEEE Access, vol. 8, pp. 24914-24926, 2020.

[28] H. Yang, K. Zheng, L. Zhao, and L. Hanzo, "Twin-timescale radio resource management for ultra-reliable and low-latency vehicular networks," IEEE Transactions on Vehicular Technology, vol. 69, no. 1, pp. 1023-1036, January 2020.

[29] S. Samarakoon, M. Bennis, W. Saad, and M. Debbah, "Distributed federated learning for ultra-reliable low-latency vehicular communications," IEEE Transactions on Communications, vol. 68, no. 2, pp. 1146-1159, February 2020.

[30] C. Xiao et al., "Downlink MIMO-NOMA for ultra-reliable low-latency communications," IEEE Journal on Selected Areas in Communications, vol. 37, no. 4, pp. 780-794, April 2019.

[31] M. Amjad and L. Musavian, "Performance analysis of NOMA for ultrareliable and low-latency communications," in 2018 IEEE Globecom Workshops (GC Wkshps), December 2018, pp. 1-5.
[32] R. Kotaba et al., "Improving spectral efficiency in URLLC via NOMABased retransmissions," in ICC 2019 - 2019 IEEE International Conference on Communications (ICC), May 2019, pp. 1-7.

[33] X. Sun et al., "Short-packet downlink transmission with non-orthogonal multiple access," IEEE Transactions on Wireless Communications, vol. 17 , no. 7, pp. 4550-4564, July 2018.

[34] A. Azari, M. Ozger, and C. Cavdar, "Risk-aware resource allocation for URLLC: Challenges and strategies with machine learning," IEEE Communications Magazine, vol. 57, no. 3, pp. 42-48, March 2019.

[35] R. M. Cuevas, C. Rosa, F. Frederiksen, and K. I. Pedersen, "Uplink ultra-reliable low-latency communications assessment in unlicensed spectrum," in 2018 IEEE Globecom Workshops, December 2018, pp. $1-6$.

[36] C. Liu, M. Bennis, M. Debbah, and H. V. Poor, "Dynamic task offloading and resource allocation for ultra-reliable low-latency edge computing," IEEE Transactions on Communications, vol. 67, no. 6, pp. 4132-4150, 2019.

[37] L. Zhang and Y. Liang, "Average throughput analysis and optimization in cooperative IoT networks with short packet communication," IEEE Transactions on Vehicular Technology, vol. 67, no. 12, pp. 11549 11 562, December 2018.

[38] Y. Hu, M. Serror, K. Wehrle, and J. Gross, "Finite blocklength performance of cooperative multi-terminal wireless industrial networks," IEEE Transactions on Vehicular Technology, vol. 67, no. 7, pp. 5778-5792, July 2018.

[39] Y. Hu, A. Schmeink, and J. Gross, "Optimal scheduling of reliabilityconstrained relaying system under outdated CSI in the finite blocklength regime," IEEE Transactions on Vehicular Technology, vol. 67, no. 7, pp. 6146-6155, July 2018.

[40] P. Nouri, H. Alves, and M. Latva-aho, "Performance analysis of ultra-reliable short message decode and forward relaying protocols," EURASIP Journal on Wireless Communications and Networking, vol. 2018, no. 1, p. 202, August 2018.

[41] Y. Gu, H. Chen, Y. Li, and B. Vucetic, "Ultra-reliable short-packet communications: Half-duplex or full-duplex relaying?" IEEE Wireless Communications Letters, vol. 7, no. 3, pp. 348-351, June 2018

[42] G. Durisi, T. Koch, and P. Popovski, "Toward massive, ultra-reliable, and low-latency wireless communication with short packets," Proceedings of the IEEE, vol. 104, no. 9, pp. 1711-1726, September. 2016.

[43] C. She, C. Yang, and T. Q. S. Quek, "Radio resource management for ultra-reliable and low-latency communications," IEEE Communications Magazine, vol. 55, no. 6, pp. 72-78, June 2017.

[44] Y. Polyanskiy, H. V. Poor, and S. Verdu, "Channel coding rate in the finite blocklength regime," IEEE Transactions on Information Theory, vol. 56, no. 5, pp. 2307-2359, May 2010.

[45] W. Yang, G. Durisi, T. Koch, and Y. Polyanskiy, "Quasi-static multipleantenna fading channels at finite blocklength," IEEE Transactions on Information Theory, vol. 60, no. 7, pp. 4232-4265, July 2014.

[46] P. Popovski et al., "Wireless access for ultra-reliable low-latency communication: Principles and building blocks," IEEE Network, vol. 32, no. 2, pp. 16-23, March 2018

[47] Y. Hu, M. Ozmen, M. C. Gursoy, and A. Schmeink, "Optimal power allocation for QoS-constrained downlink multi-user networks in the finite blocklength regime," IEEE Transactions on Wireless Communications, vol. 17, no. 9, pp. 5827-5840, September 2018.

[48] W. R. Ghanem, V. Jamali, Y. Sun, and R. Schober, "Resource allocation for multi-user downlink URLLC-OFDMA systems," in 2019 IEEE International Conference on Communications Workshops (ICC Workshops), 2019, pp. 1-6.

[49] A. Anand and G. de Veciana, "Resource allocation and HARQ optimization for URLLC traffic in 5G wireless networks," IEEE Journal on Selected Areas in Communications, vol. 36, no. 11, pp. 2411-2421, November 2018.

[50] C. She, C. Yang, and T. Q. S. Quek, "Cross-layer optimization for ultrareliable and low-latency radio access networks," IEEE Transactions on Wireless Communications, vol. 17, no. 1, pp. 127-141, January 2018.

[51] — , "Joint uplink and downlink resource configuration for ultrareliable and low-latency communications," IEEE Transactions on Communications, vol. 66, no. 5, pp. 2266-2280, May 2018

[52] B. Makki, T. Svensson, and M. Zorzi, "Finite block-length analysis of the incremental redundancy HARQ," IEEE Wireless Communications Letters, vol. 3, no. 5, pp. 529-532, October 2014.

[53] E. Dosti, M. Shehab, H. Alves, and M. Latva-aho, "Ultra reliable communication via CC-HARQ in finite block-length," in 2017 European Conference on Networks and Communications (EuCNC), June 2017, pp. $1-5$. 
[54] J. P. Battistella Nadas et al., "Performance analysis of hybrid ARQ for ultra-reliable low latency communications," IEEE Sensors Journal, vol. 19, no. 9, pp. 3521-3531, 2019.

[55] A. Avranas, M. Kountouris, and P. Ciblat, "Energy-latency tradeoff in ultra-reliable low-latency communication with retransmissions," IEEE Journal on Selected Areas in Communications, vol. 36, no. 11, pp. 24752485, November 2018.

[56] H. Shariatmadari et al., "Resource allocations for ultra-reliable lowlatency communications," International Journal of Wireless Information Networks, vol. 24, no. 3, pp. 317-327, September 2017.

[57] C. Sun, C. She, and C. Yang, "Retransmission policy with frequency hopping for ultra-reliable and low-latency communications," in 2018 IEEE International Conference on Communications (ICC), May 2018 pp. $1-6$.

[58] 3GPP Technical Documents R1-1808449, "IMT-2020 self-evaluation: UP latency analysis for FDD and dynamic TDD with UE processing capability 2 (URLLC)," August 2018.

[59] H. Shariatmadari et al., "Fifth-Generation control channel design: Achieving ultra-reliable low-latency communications," IEEE Vehicular Technology Magazine, vol. 13, no. 2, pp. 84-93, June 2018.

[60] G. Ku and J. M. Walsh, "Resource allocation and link adaptation in LTE and LTE-Advanced: A tutorial," IEEE Communications Surveys Tutorials, vol. 17, no. 3, pp. 1605-1633, third-quarter 2015.

[61] P. Popovski et al., "Wireless access in ultra-reliable low-latency communication (URLLC)," IEEE Transactions on Communications, vol. 67, no. 8, pp. 5783-5801, 2019.

[62] G. Pocovi, K. I. Pedersen, and P. Mogensen, "Joint link adaptation and scheduling for $5 \mathrm{G}$ ultra-reliable low-latency communications," IEEE Access, vol. 6, pp. 28912-28 922, 2018.

[63] A. Karimi, K. I. Pedersen, and P. Mogensen, "Low-complexity centralized multi-cell radio resource allocation for 5G URLLC," in 2020 IEEE Wireless Communications and Networking Conference (WCNC), 2020, pp. 1-6.

[64] G. Pocovi et al., "Achieving ultra-reliable low-latency communications: Challenges and envisioned system enhancements," IEEE Network, vol. 32, no. 2, pp. 8-15, March 2018.

[65] H. Shariatmadari et al., "Statistical analysis of downlink transmissions for ultra-reliable low-latency communications," in 2018 15th International Symposium on Wireless Communication Systems (ISWCS), August 2018, pp. $1-5$

[66] _ "Optimized transmission and resource allocation strategies for ultra-reliable communications," in 2016 IEEE 27th Annual International Symposium on Personal, Indoor, and Mobile Radio Communications (PIMRC), September 2016, pp. 1-6.

[67] G. Berardinelli et al., "On the benefits of early HARQ feedback with non-ideal prediction in 5G networks," in 2016 International Symposium on Wireless Communication Systems (ISWCS), September 2016, pp. 1115

[68] B. Makki, T. Svensson, G. Caire, and M. Zorzi, "Fast HARQ over finite blocklength codes: A technique for low-latency reliable communication,' IEEE Transactions on Wireless Communications, vol. 18, no. 1, pp. 194209, January 2019.

[69] K. Pedersen, G. Pocovi, J. Steiner, and A. Maeder, "Agile 5G scheduler for improved E2E performance and flexibility for different network implementations," IEEE Communications Magazine, vol. 56, no. 3, pp. 210-217, March 2018.

[70] P. Popovski, "Ultra-reliable communication in 5G wireless systems," in 1st International Conference on $5 G$ for Ubiquitous Connectivity, November 2014, pp. 146-151.

[71] J. Chen et al., "Resource allocation for wireless-powered IoT networks with short packet communication," IEEE Transactions on Wireless Communications, vol. 18, no. 2, pp. 1447-1461, February 2019.

[72] C. Sun et al., "Optimizing resource allocation in the short blocklength regime for ultra-reliable and low-latency communications," IEEE Transactions on Wireless Communications, vol. 18, no. 1, pp. 402-415, January 2019.

[73] J. Zeng et al., "Achieving ultra-reliable and low-latency communications in IoT by FD-SCMA," IEEE Internet of Things Journal, vol. 7, no. 1, pp. 363-378, January 2020

[74] - "Enabling ultrareliable and low-latency communications under shadow fading by massive MU-MIMO," IEEE Internet of Things Journal, vol. 7, no. 1, pp. 234-246, January 2020.
[75] J. Scarlett, V. Y. F. Tan, and G. Durisi, "The dispersion of nearestneighbor decoding for additive non-gaussian channels," IEEE Transactions on Information Theory, vol. 63, no. 1, pp. 81-92, January 2017.

[76] J. Park, "Rate analysis of ultra-reliable low-latency communications in random wireless networks," arXiv e-prints, p. arXiv:1910.13868, October 2019

[77] N. H. Mahmood et al., "On the resource utilization of multi-connectivity transmission for URLLC services in 5G New Radio," in 2019 IEEE Wireless Communications and Networking Conference Workshop (WCNCW), 2019, pp. 1-6.

[78] K. I. Pedersen, S. R. Khosravirad, G. Berardinelli, and F. Frederiksen, "Rethink hybrid automatic repeat request design for 5G: Five configurable enhancements," IEEE Wireless Communications Magazine, vol. 24, no. 6, pp. 154-160, December 2017.

[79] H. Shariatmadari et al., "Asymmetric ACK/NACK detection for ultrareliable low-latency communications," in 2018 European Conference on Networks and Communications (EuCNC), June 2018, pp. 1-166.

[80] S. Boyd, S. Kim, L. Vandenberghe, and A. Hassibi, "A tutorial on geometric programming," Optimization and Engineering, vol. 8, no. 1, pp. 67-127, April 2007.

[81] Y. Sun, D. W. K. Ng, Z. Ding, and R. Schober, "Optimal joint power and sub-carrier allocation for full-duplex multi-carrier non-orthogonal multiple access systems," IEEE Transactions on Communications, vol. 65 , no. 3, pp. 1077-1091, March 2017.

[82] C. Condo, S. Ali Hashemi, and W. J. Gross, "Blind detection with polar codes," IEEE Communications Letters, vol. 21, no. 12, pp. 2550-2553, 2017.

[83] A. Balatsoukas-Stimming and A. Filos-Ratsikas, "On the computational complexity of blind detection of binary linear codes," in 2019 IEEE International Symposium on Information Theory (ISIT), 2019, pp. 24492453.

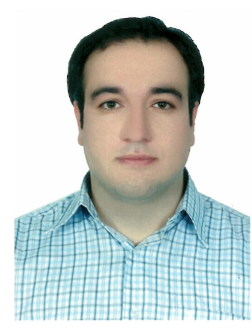

Ali Karimidehkordi received his B.Sc. in electrical engineering-electronics from Isfahan University of Technology, Iran, and his M.Sc. degree in electrical engineering-communication systems from the University of Tehran, Iran. In 2020, he received his $\mathrm{Ph} . \mathrm{D}$. in wireless communications from Aalborg University, in close collaboration with Nokia Bell Labs, Denmark. His main Ph.D. focus was on multiservice radio resource management for $5 \mathrm{G}$ networks and he was part of EU funded projects ONE5G and FANTASTIC-5G. He is currently working as Device Research Specialist with Nokia Bell Labs, Munich, Germany. His main research interests are $5 \mathrm{G}$ device/network performance optimization, ultrareliable low-latency communications (URLLC), and operation through the millimeter-wave spectrum.

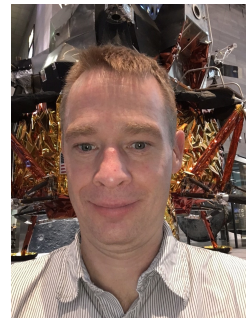

Klaus I. Pedersen (Senior Member, IEEE) received the M.Sc. degree in electrical engineering and the Ph.D. degree from Aalborg University, Aalborg, Denmark, in 1996 and 2000, respectively. He is currently leading the Nokia Bell Labs radio access systems research team in Aalborg, and a part-time Professor with the Wireless Communications Network (WCN) Section, Aalborg University. He is the author or coauthor of approximately 200 peerreviewed publications on a wide range of topics, as well as inventor on several patents. His current work is related to $5 \mathrm{G}$ new radio evolution, including radio resource management aspects to enable new use cases with special emphasis on mechanisms that offer improved end-to-end (E2E) performance delivery. He was recently also part of the EU funded research project ONE5G that focused on E2E-aware Optimizations and advancements for the Network Edge of 5G New Radio that was successfully concluded in the Summer 2019. 


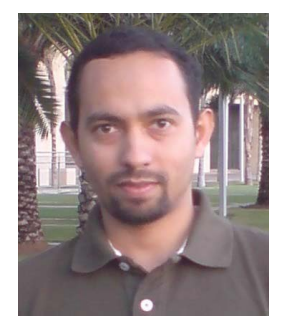

Nurul Huda Mahmood (M'13) was born in Bangladesh. He received the Ph.D. degree in wireless communications from Norwegian University of Science and Technology (NTNU), Norway in 2012. Currently, Nurul is a Senior Research Fellow with 6G Flagship at Center for Wireless Communication, University of Oulu. Prior to that, he was an Associate Professor with Department of Electronics Systems at Aalborg University, Denmark. His current research interests include resource optimization techniques with focus on ultra-reliable low-latency communications (URLLC), critical internet of things (IoT) networks, and modelling and performance analysis of wireless communication systems.

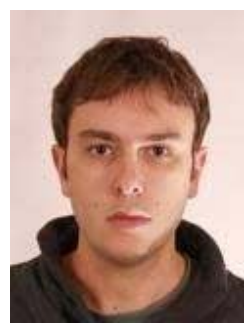

Gilberto Berardinelli received the first and second level degrees (cum laude) in telecommunication engineering from the University of LAquila, Italy, in 2003 and 2005, respectively, and the Ph.D. degree from Aalborg University, Denmark, in 2010. He is currently an Associate Professor with the Wireless Communication Networks (WCN) Section, Aalborg University, and also working in tight cooperation with Nokia Bell Labs. His research interests include mostly focused on physical layer, medium access control, and radio resource management design for $5 \mathrm{G}$ systems and beyond. He is the author or coauthor of more than 100 international publications, including conference proceedings, journal contributions, and book chapters.

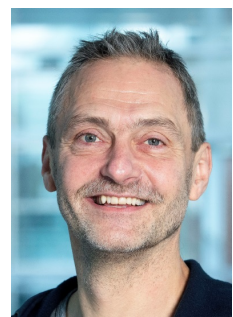

Preben Mogensen received the M.Sc. and Ph.D degrees from Aalborg University, in 1988 and 1996, respectively. Since 1995 , he has been part-time associated with Nokia Bell Labs. Since 2000, he has been a Full Professor with Aalborg University, where he is currently leading the Wireless Communication Networks Section, Department of Electronic Systems. $\mathrm{He}$ is also a Principal Scientist with Nokia Bell Labs. $\mathrm{He}$ has co-authored more than 400 articles in various domains of wireless communication. His current research interest includes $5 \mathrm{G}$ evolution towards $6 \mathrm{G}$. 